\title{
Editorial
}

\section{Tétrade de Fallot: Qual o Real Benefício da Correção Operatória na Idade Adulta?}

\author{
Edmar Atik, Fernando Antibas Atik \\ São Paulo, SP
}

Apesar da raridade de pacientes com tétrade de Fallot clássica, que atingem a idade adulta sem correção total, correspondendo cerca de $12 \%$ com 20 anos e apenas a $2 \%$ com 40 anos $^{1}$, cresce a importância do conhecimento da sua apresentação clínica, fisiopatológica e evolutiva pós-cirúrgica, em face do aumento progressivo de casos de cardiopatias congênitas em geral, nesta faixa etária.

Preliminarmente pressupõe-se que a anatomia da tétrade de Fallot no adulto seja mais favorável do que a encontrada na criança. Pois, em face da chamada lei da seleção natural dos casos patológicos, os que evoluem por maior período de tempo guardam uma relação direta com melhores condições anatômicas, constituindo número menor de pacientes com tétrade de Fallot que naturalmente alcança a $3^{\circ}$ década da vida.

No entanto, funcionalmente tendem a mostrar deterioração dada a presença de vários fatores na evolução e até de complicações que inclusive interferem na evolução pós-operatória tardia, apresentando-se como co-morbidades ${ }^{2-6}$.

São eles representados pela hipóxia crônica, a policitemia, a hipertrofia miocárdica, alterações da coagulação, disfunção miocárdica secundária, consequências morfológicas e funcionais de anastomoses prévias e complicações e intercorrências como abscesso cerebral, endocardite infecciosa, embolias, tromboses e arritmias.

Assim, se por um lado a anatomia é mais favorável, a função passa a ser mais deteriorada suscitando dúvidas daí quanto ao real benefício da correção operatória nesta faixa etária.

De uma coletânea dos principais trabalhos relatados na literatura ${ }^{2-6}$ sobre reparo cirúrgico da tétrade de Fallot no adulto (tab. 1), em 493 pacientes observou-se presença de uma anatomia realmente mais favorável dado que no reparo da obstrução da via de saída do ventrículo direito o anel pulmonar foi ampliado em 204 pacientes $(41,3 \%)$, alargamento das artérias pulmonares feito em $22(4,4 \%)$ e a colocação de homoenxerto em $8(1,6 \%)$ o que correspondem a percentagens bem menores de procedimentos das que ocorrem na criança em geral.

Ademais, operações prévias anastomóticas sistêmico-pulmonares, principalmente tipo Blalock-Taussig foram realizadas em 151 pacientes, 30,6\% do total, o que também é realizado em número maior de casos em crianças.

Instituto do Coração do Hospital das Clínicas da Faculdade de Medicina da Universidade de São Paulo

Endereço para Correspondência: Edmar Atik - InCor - Hospital das Clínicas - FMUSP - Av Eneas Carvalho de Aguiar 44

Cep 05403-000 - São Paulo - SP - E-mail: conatik@incor.usp.br Recebido para Publicação em 09/04/2004
Intercorrências encontradas em 83 pacientes, correspondendo a $16,8 \%$ do total em período prévio à operação no adulto, como endocardite infecciosa em 13 pacientes, abscessos cerebrais em 18 , hemoptises em 36 , trombose cerebral em 12 e tuberculose pulmonar em 4 , ocorrem em número maior também nessa faixa etária do que nas crianças, o que pode interferir na evolução pósoperatória a longo prazo.

Na evolução pós-operatória, reoperações são mais raras que na criança, em especial a comunicação interventricular realizada em 12, estenose pulmonar em 11, insuficiência tricúspide em 2, insuficiência pulmonar em um. Assim, reintervenções foram necessárias em 29 pacientes, correspondendo a 6,6\% do total dos operados na literatura, o que se constitui em outro elemento que confirma a anatomia mais favorável neste grupo etário, dado o menor número de casos reoperados.

A melhor evolução com a operação, avaliada em 194 pacientes $^{2-6}$ através a classe funcional I e II em 189 (97,4\%) dos casos em relação a $99(50,9 \%)$ nestas mesmas classes no período préoperatório, atesta sua efetividade.

As mortalidades, imediata e tardia, mais acentuadas que na criança, em 54 dos 493 (10,9\%) e em 53 dos 439 (9,8\%) respectivamente, decorrem do aspecto funcional deteriorado.

Na experiência do grupo cirúrgico do InCor-FMUSP7, em 39 pacientes adultos operados com tétrade de Fallot, de novembro 1982 a janeiro 2001, com idades entre 18 a 67 (média de 26 $+11,1$ ) anos, verificaram-se os mesmos achados encontrados na literatura em relação aos aspectos anatomofuncionais, com algumas variações. Nesse material, operações prévias foram realizadas em apenas 4 (10\%) pacientes, em período de 16 a 45 anos antes da correção, com média de 25 anos, o remendo transanular pulmonar foi necessário em 6 (15,4\%), o alargamento da via de saída de ventrículo direito em 17 (43,6\%). A incisão de ventrículo direito foi necessária em 23 (59\%) pacientes, para a ressecção de bandas musculares e fechamento da comunicação interventricular, a comissurotomia pulmonar em 13 (33,3\%), a substituição da valva pulmonar por bioprótese em $3(7,7 \%)$ e outros procedimentos associados, como reparo de comunicação interatrial, canal arterial, drenagem anômala parcial das veias pulmonares, fístula coronário-cavitária e das valvas mitral, tricúspide e aórtica em 8 (20,5\%). As complicações pré-operatórias, como endocardite infecciosa em 1 , hemoptise em 2 , acidente vascular cerebral em 4 e síncope em 9 incidiram em maior número, em 16 (41\%) dos casos. E houve associação a outras doenças em $10 \%$ dos casos, como diabetes, insuficiência coronariana obstrutiva, cirrose hepática e hipotiroidismo. 


\begin{tabular}{|c|c|c|}
\hline \multicolumn{3}{|c|}{$\begin{array}{c}\text { Tabela I - Dados da literatura }{ }^{2-6} \text { acerca do reparo cirúrgico da } \\
\text { tetralogia de Fallot no adulto }\end{array}$} \\
\hline & $\mathrm{N}$ & $(\%)$ \\
\hline Número de pacientes & 493 & \\
\hline Operações paliativas prévias & 151 & 30,6 \\
\hline Blalock-Taussig & 139 & 28,2 \\
\hline Brock & 11 & 2,2 \\
\hline Waterston Cooley & 1 & 0,2 \\
\hline Doenças prévias & 83 & 16,8 \\
\hline Endocardite infecciosa & 13 & \\
\hline Abscesso cerebral & 18 & \\
\hline Trombose cerebral & 12 & \\
\hline Hemoptise & 36 & \\
\hline Tuberculose pulmonar & 4 & \\
\hline \multicolumn{3}{|l|}{ Técnicas operatórias } \\
\hline Remendo transanular pulmonar & 204 & 41,3 \\
\hline Alargamento das artérias pulmonares & 22 & 4,4 \\
\hline Homoenxerto & 8 & 1,6 \\
\hline Mortalidade hospitalar* & 54 & 10,9 \\
\hline Mortalidade tardia* & 43 & 9,8 \\
\hline Reoperações & 29 & 6,6 \\
\hline Comunicação interventricular & 12 & \\
\hline Estenose pulmonar & 11 & \\
\hline Insuficiência pulmonar & 1 & \\
\hline Insuficiência tricúspide & 2 & \\
\hline Aneurisma da aorta & 1 & \\
\hline Homoenxerto & 2 & \\
\hline Complicações tardias & 17 & 3,8 \\
\hline Arritmias & 12 & \\
\hline Insuficiência cardíaca & 5 & \\
\hline \multicolumn{3}{|c|}{$\begin{array}{l}\text { * Causas principais: baixo débito cardíaco, hemorragia pulmonar, insu- } \\
\text { ficiência cardíaca, morte súbita, distúrbios de coagulação, insuficiência } \\
\text { de múltiplos órgãos, endocardite infecciosa. }\end{array}$} \\
\hline
\end{tabular}

A fim de se ter noção mais acurada do aspecto funcional no grupo operado no InCor-FMUSP ${ }^{7}$, a saturação arterial de oxigênio pré-operatória variou de 56 a 95\% com média de $84,8+9,9 \%$. 0 hematócrito variou de 35 a $75 \%$ com média de $53,6+10 \%$.

A evolução imediata pós-operatória e a recuperação hospitalar até a alta ocorreram sem problemas com suporte inotrópico usual com dopamina, dobutamina e milrinone na maioria dos nossos pacientes tendo eles obtido alta da UTI em média após 2 dias e do hospital em média após 8 dias, com mortalidade imediata de $3(7,6 \%)$ pacientes.

Na evolução tardia, em média de 45,1+54,5 meses, variando de 1 a 194 meses, constatou-se que a maioria estava em classe funcional I e II (33 dos 34 pacientes) em relação ao encontrado antes da operação no qual predominavam as classes II e III (36 dos 39 pacientes), semelhante à encontrada na literatura.

Por deterioração da função ventricular a maior prazo por todas as possíveis razões expostas e relacionadas principalmente a aspectos pré-operatórios nota-se diminuição da sobrevida a maior prazo através a curva atuarial de $91,2 \%$ em 3 anos, $85,5 \%$ em 7 anos, $68,4 \%$ em 15 anos, superponível às também relatadas na literatura. Deve-se tal evolução ao aparecimento de arritmias e de insuficiência cardíaca no decurso do tempo.

Esse aspecto foi bem salientado por Presbitero e cols. ${ }^{21}$, demonstrando evolução desfavorável após a operação a maior prazo. Assim, esses autores mostraram que nos primeiros anos após a correção, estando a maioria dos pacientes em classe funcional I, em 30-76,9\%, e II em 8-20,5\% após 1 a 11 anos da operação em média de 3 anos, decresce com maior tempo de evolução para cerca de $16-45,7 \%$ em classe funcional I, $18-51,4 \%$ em II e 12,8\% em III após 10 a 20 anos, com média de 13 anos. A classe funcional prévia à operação era em $23-57,5 \%$ em II, 14-35\% em III e 3-7,5\% em IV,dentre 40 pacientes operados de 20 a 67 anos.

No entanto, outros referem evolução favorável após a operação, relatada por Nollert $\mathrm{G}$ e cols. ${ }^{8}$ apresentando semelhante curva atuarial da apresentada pela população geral. Este fato salienta, mesmo com outras experiências menos favoráveis, de que a operação na idade adulta é factível e indicada apesar da co-morbidade dos fatores adquiridos, a maior prazo.

Na tétrade de Fallot, é preferível a intervenção mais precoce, mas os resultados da correção na idade adulta não são desanimadores a ponto dos pacientes nessa faixa etária não serem considerados já ultrapassados à obtenção de uma vida melhor através a intervenção cirúrgica, mesmo que tardia ${ }^{9,10}$.

\section{Referências}

1. Kirklin JW, Barratt-Boyes BG. Cardiac Surgery: Morphology, Diagnostic Criteria, Natural History, Techniques, Results, and Indications. 2nd ed. New York: Churchill Livingstone, 1993.

2. Dittrich S, Vogel M, Dahnert I, et al. Surgical repair of tetralogy of Fallot in adults today. Clin Cardiol. 1999; 22:460-4.

3. Presbitero P, Demarie D, Aruta E, et al. Results of total correction of tetralogy of Fallot performed in adults. Ann Thorac Surg. 1988; 46:297-301.

4. Rammohan M, Airan B, Bhan A, et al. Total correction of tetralogy of Fallot in adults-surgical experience. Int J Cardiol. 1998; 63:121-8.

5. Hu DC, Seward JB, Puga FJ, et al. Total correction of tetralogy of Fallot at age 40 years and older: long-term follow-up. J Am Coll Cardiol. 1985; 5:40-4.

6. Presbitero P, Prever SB, Contrafatto I, Morea M. As originally published in 1988: Results of total correction of tetralogy of Fallot performed in adults. Updated in 1996. Ann Thorac Surg. 1996; 61:1870-3.
7. Atik FA, Atik E, Cunha CR, et al. Long-term results of correction of tetralogy of Fallot in adulthood. EJCTS. 2004;25: 250-255.

8. Nollert G, Fischlein T, Bouterwek S, et al. Long-term results of total repair of tetralogy of Fallot in adulthood: 35 years follow-up in 104 patients corrected at the age of 18 or older. Thorac Cardiovasc Surg. 1997; 45:178-81.

9. Vliegen HW, van Straten A, de Roos A, et al. Magnetic resonance imaging to assess the hemodynamic effects of pulmonary valve replacement in adults late after repair of tetralogy of fallot. Circulation. 2002; 106:1703-7.

10. Davlouros PA, Kilner PJ, Hornung TS, et al. Right ventricular function in adults with repaired tetralogy of Fallot assessed with cardiovascular magnetic resonance imaging: detrimental role of right ventricular outflow aneurysms or akinesia and adverse right-to-left ventricular interaction. J Am Coll Cardiol. 2002; 40:2044-52. 